\title{
Energy sensitive X-ray phase contrast imaging with a CdTe-Timepix3 detector
}

\author{
C. Navarrete, S. Procz, J. Fey, G. Roque, C. Avila, M. Schuetz, A. Olivo and M. Fiederle
}

\begin{abstract}
The Timepix 3 is a photon counting semiconductor detector that enables to simultaneously measure the energy and time of arrival of each incident $X$ - ray photon. These properties, along with the high spatial resolution and high efficiency, due to the CdTe sensor material, can be exploited for several imaging applications, such as X-ray phase contrast imaging (XPCI).

XPCI relies on the phase shift suffered by $\mathrm{X}$-rays when traversing the sample. This study focuses on the free-space propagation XPCI and single mask edge illumination XPCI methods, which are two approaches that are well suited for laboratory implementations.

Since both techniques are highly sensitive to charge-sharing, the Timepix 3 energy and time information for each photon are used to minimize this effect by using pixel clustering methods. In addition, the performance of both XPCI techniques across a $30 \mathrm{kVp}$ source spectrum is studied using the energy-resolving capabilities of the detector. In both cases, the phase contrast and signal-to-noise ratio (SNR) are assessed as a function of different energy. Finally, it is demonstrated that phase contrast enhancement is feasible with pixel clustering and energy-selection for both XPCI techniques.
\end{abstract}

\section{INTRODUCTION}

\section{A. X-ray phase contrast imaging}

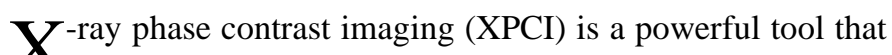
$\chi_{\text {can }}$ be exploited for several applications, including biomedical imaging [1]-[3], security [4], [5] and material science [6], [7]. It relies on the phase shift suffered by X-rays when traversing the sample, which is related to the unit decrement of the real part of the complex refractive index. Conversely, the imaginary term is responsible for the attenuation properties. Since the real coefficient can be up to three orders magnitude greater than the complex one, depending on the incident X-ray spectrum, XPCI techniques allow the visualization of details previously considered invisible to conventional absorption radiography [8].

Different phase-sensitive methods have been developed in order to use the XPCI principle. Among them, free-space

Manuscript received December 13, 2019. This work was supported the Physics Department and the Faculty of Science of Universidad de los Andes under project number P19.160322.002-20FISI01, by COLCIENCIAS under contract number 80740-055-2019 and by the German Ministry of Research and Education (BMBF) under project MAMMOSECUR (Förderkennzeichen 01DN19002).

C.Navarrete, G. Roque and C.Avila are with the Physics Department of Universidad de los Andes, Cra $1 \mathrm{~N}^{\circ} 18 \mathrm{~A}-12,111711$, Bogotá, Colombia (email: cf.navarrete969@uniandes.edu.co).

S. Procz, J. Fey, M. Schuetz and M. Fiederle are with the Freiburg Materials Research Center (FMF), Stefan-Meier-Str. 21, 79104 Freiburg, Germany (e-mail: simon.procz@fmf.uni-freiburg.de).

A. Olivo is with the Department of Medical Physics and Biomedical engineering, University College London, Malet Place, Gower Street, WC1E 6BT London, United Kingdom (e-mail: a.olivo@ucl.ac.uk) propagation and single mask edge illumination (SM-EI) are two approaches that are well suited for laboratory implementations.

\section{B. Free-space propagation (FSP) XPCI}

Free-space propagation XPCI, also called in-line XPCI, was first introduced with monochromatic hard X-rays at synchrotron sources by Snigirev et al. [9], who demonstrated that phase effects can be observed with a source of sufficient spatial coherence and by placing the detector at a distance from the sample such that the perturbed and unperturbed parts of the wave can interfere (see Fig. 1a). Later, Wilkins et al. [10] extended the in-line XPCI to polychromatic laboratory sources and subsequent studies showed the potential of this technique for imaging of weakly absorbing samples [11], [12]. The method has been widely implemented for synchrotron [13], [14] and lab-based [15], [16] imaging and tomography, however, due to its high lateral spatial coherence requirements, only microfocus laboratory sources have shown significant phase signals.

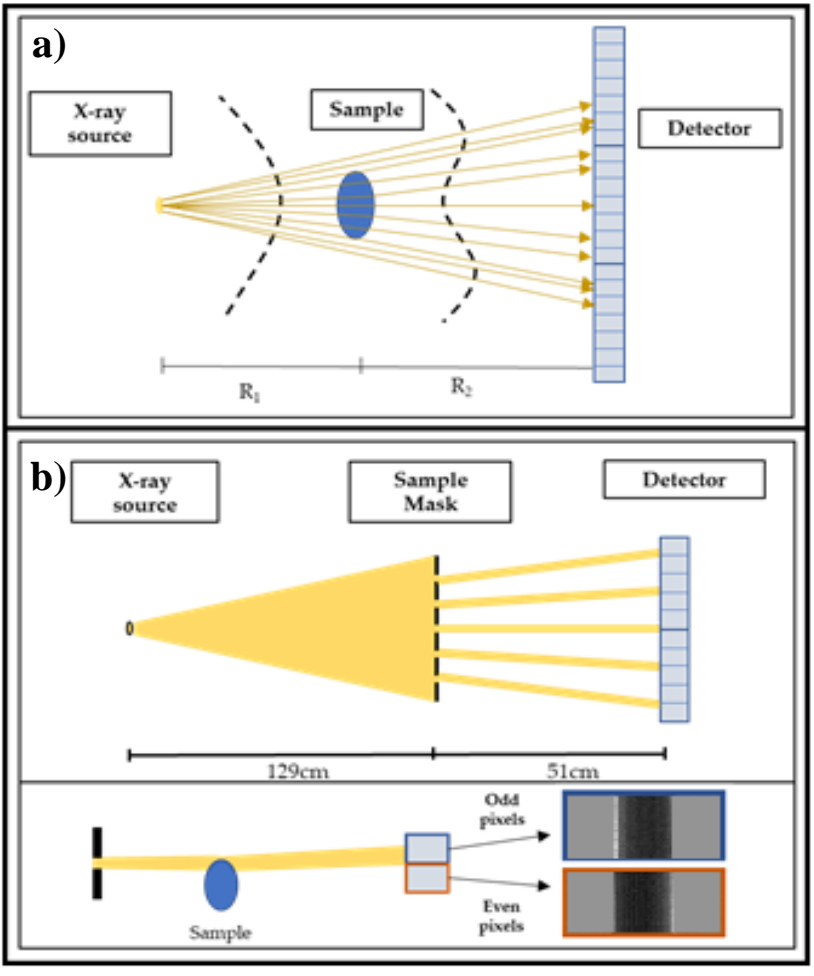

Fig. 1. a) Schematic and working principle of free-space propagation XPCI. The incoming wave is perturbed by the sample and the detector is placed sufficiently distant, so an interference is produced. b) Schematic and working principle of single mask edge illumination XPCI. The mask apertures define beamlets which are aligned with the pixel boundaries. Odd and even pixels can be separated to obtain two images with opposite refraction signals. 


\section{MATERIALS AND METHODS}

\section{Single mask edge illumination (SM-EI) XPCI}

SM-EI XPCI is based on the edge-illumination principle, originally described by Olivo et al [17], in which the incoming beam is shaped in smaller beamlets by a periodic mask that is placed before the sample. In the original set-up, a second mask is placed before the detector in order to create the edgeillumination condition, which is obtained by aligning the edges of the mask so the beamlets partially impinge on the detector pixels [18]. With recent high-resolution photoncounting detector technologies with a more defined Point Spread Function (like the Medipix and Timepix families), the set-up can be simplified by removing the second mask and instead aligning the beamlets with the boundary between two pixels in order to detect the beam displacement induced by the sample (see Figure 1b) [19], [20]. By separating the odd and even pixel columns (or rows depending on the mask alignment), this arrangement allows to obtain two images with equivalent absorption information but opposite differential phase contrast signals, which effectively permits single-image phase-retrieval. Despite resulting in a loss of resolution by a factor of 2 as compared with its double-mask counterpart, this approach can provide similar phase sensitivity with twice as much statistics or half the exposure time [21].

\section{The Timepix 3 detector}

The Timepix 3 is a pixelated photon counting semiconductor detector fabricated in a CMOS $130 \mathrm{~nm}$ process and has $256 \times 256$ pixels with a pixel pitch of $55 \mu \mathrm{m}$. It extends the functionality of its predecessor of the Timepix family, by allowing simultaneous time-of-arrival (ToA) and time-overthreshold (ToT) measurements [22]. This means, simultaneous acquisition of time and energy information of each incident photon. The electronics of the Timepix 3 chip can be bump bonded to diverse sensor materials such as $\mathrm{Si}, \mathrm{CdTe}, \mathrm{CdZnTe}$, $\mathrm{Ge}$, or GaAs. Among them, CdTe is a semiconductor with high atomic number, high density, wide energy band gap and high resistivity. These features make it suitable for room temperature operation with high detection efficiency for Xrays [23].

The spectral capabilities of photon counting detectors, such as the Timepix 3 , were recently incorporated to enhance the features of different phase-sensitive methods. Das et al., used the Medipix detector for single-shot phase and absorption retrieval by separating the photons into multiple energy bins, using both the free-space propagation [24] and the double mask edge illumination techniques [25]. Later, the same was derived and simulated by Wang et al. with the grating interferometric technique [26].

In this work, the time resolution and energy resolving capabilities of the Timepix 3 detector are used to study contrast and signal-to-noise ratio (SNR) in two XPCI methods. The relevance of pixel clustering methods to correct for charge sharing effects (see section 2.4) is presented and energywindow selection is implemented to increase image quality.

\section{A. The experimental set-up}

The X-ray set-up, used in this work, consists of a

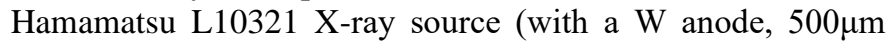
thick beryllium output window and a nominal focal spot FWHM of $5 \mu \mathrm{m}$ ), a CdTe-Timepix 3 detector and a set of high precision step motors (accuracy of $1 \mu \mathrm{m}$ ) to position the sample.

For the SM-EI XPCI set-up, the sample mask is made of gold with nominal thickness of $100 \mu \mathrm{m}$, electroplated on $500 \mu \mathrm{m}$ of graphite. It has a period of $79 \mu \mathrm{m}$ and an aperture size of $10 \mu \mathrm{m}$. An additional set of high precision stepping motors (accuracy of $0.2 \mu \mathrm{m}$ and $0.001^{\circ}$ ) is required to match the projected mask and detector periods $(110 \mu \mathrm{m})$, in order to create the 'edge illumination condition' for every other pixel column.

\section{B. Verification of Timepix 3 energy calibration}

The Timepix 3 detector energy calibration, for the present studies, was initially performed by the manufacturer (Advacam) as described in [27], however, the energy response of the detector was verified again in our labs through an experimental measurement. This is obtained through X-ray fluorescence measurements of $\mathrm{Zn}, \mathrm{Rh}$ and $\mathrm{Sn}$, and the Fe55 radioactivity spectrum for low energies (see Fig. 2 top). Only events that took place in a single pixel are used for the calibration to avoid charge sharing effects.

The result of the energy calibration verification is shown in Fig. 2 bottom. The curve shows a high linearity and agreement is observed between the nominal and measured peak energies.
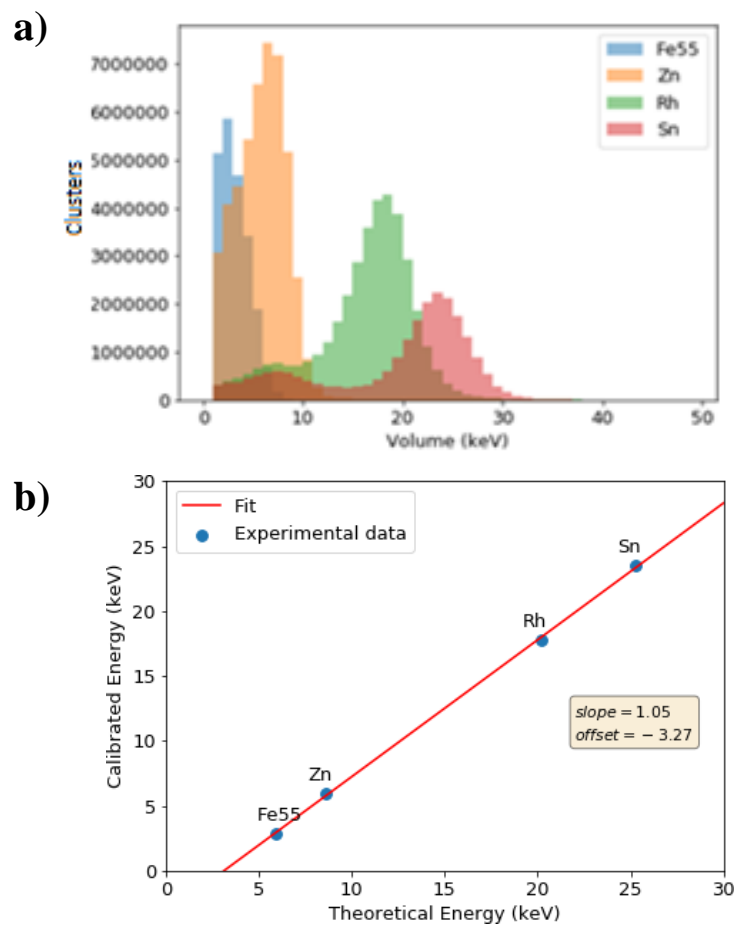

Fig. 2. a) Histograms of selected X-ray fluorescence and radioactive (Fe-55) spectrums for energy calibration verification. b) Nominal vs peak energy position. 


\section{Measurements}

All images were acquired using a nylon fiber with a diameter of $900 \mu \mathrm{m}$ at $30 \mathrm{kVp}$ for FSP XPCI and SM-EI XPCI. The clustering and energy bin analysis presented in the following sections are completed on the FSP XPCI measurement; however, certain validation aspects are based on the SM-EI XPCI measurement.

The detector is placed at $180 \mathrm{~cm}$ from the source for both XPCI techniques. The magnification value for the FSP-XPCI was $\mathrm{MFSP}=5 \mathrm{x}$ to minimize blurring caused by the focal spot on the sample edges. Conversely, for the SM-EI XPCI method, the fiber was placed very close to the sample mask at a magnification of $\mathrm{MSM}=1.3 \mathrm{x}$. All images were flat field corrected to account for any detector imperfections.

\section{Pixel clustering methods}

Charge sharing is a typical effect present in photon counting pixel detectors. It is caused by the spread of the charge cloud, generated by a single high-energy event, over adjacent pixels (see Fig. 3). Such phenomenon affects the detector spatial and energy resolution. Previous studies have shown that it increases with thicker sensor material, smaller pixel pitch and lower bias voltage [28].

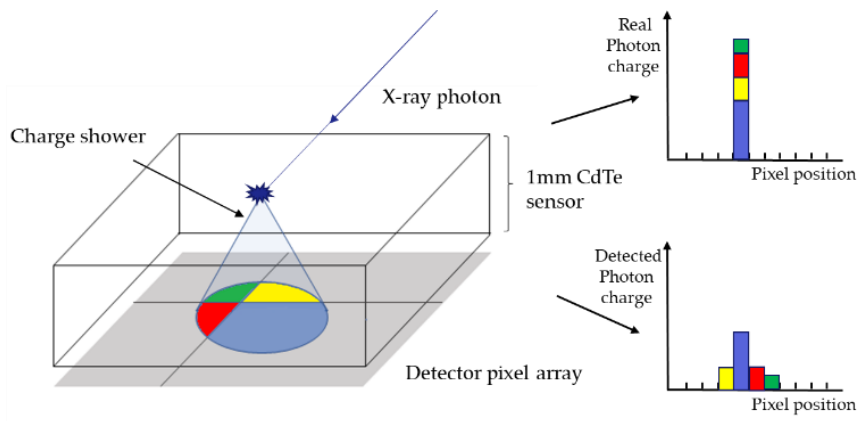

Fig. 3. Schematic of charge-sharing effect in photon-counting detectors. The charge spreads over adjacent pixels and results in multiple low charge counts.

Since the Timepix3 detector allows for energy and time-ofarrival information of each event, these data can be used to form clusters of events that originally corresponded to a single photon. Such clusters are assembled by events that are both close in space and time-of-arrival. The total energy of the cluster is then added together to reconstruct the original photon energy and the count is allocated to the pixel closer to the cluster centroid. Some imprecisions are expected since part of the charge could be lost if it does not exceed the pixel detector threshold to be considered as a count.

In this work, three different cases are compared. First, when no clustering algorithm is used which is called an 'unclustered' image and it is, in fact, the image that would be acquired by any photon-counting detector without energy and time information. Second, when only events that took place in a single pixel, i.e., with no additional charge detected close in time and space, are employed which is called single-pixel clusters. And third, when all-size clusters are utilized to reconstruct the correct photon energy with all the measured data.

\section{E. Energy-bins analysis and energy selection}

In order to understand the contribution of the spectral information to both XPCI techniques, the energy information was grouped in $5 \mathrm{keV}$ energy bin images, i.e., for the $30 \mathrm{kVp}$ acquisition, the energy bins are: $0-5 \mathrm{keV}, 5-10 \mathrm{keV}, 10-15$ $\mathrm{keV}, 15-20 \mathrm{keV}, 20-25 \mathrm{keV}$ and $25-30 \mathrm{keV}$. The energy intervals were selected such that statistics were sufficient to evaluate the contrast and SNR on each image. XPCI techniques usually generate a pair of bright and/or dark fringes at the sample edges and thus the contrast and SNR are calculated as follows,

$$
\begin{aligned}
& \text { Contrast }=\frac{I_{\max }-I_{\min }}{I_{\text {background }}} \\
& S N R=\frac{I_{\text {max }}-I_{\text {min }}}{2 \sqrt{\sigma\left(I_{\text {background }}\right)^{2}}}
\end{aligned}
$$

where $I_{\max }$ and $I_{\min }$ are the maximum and minimum signals in the nylon fiber profile, respectively, and $\sigma\left(I_{\text {background }}\right)$ is the standard deviation of the image in the background region. Contrast and SNR are calculated for each of the energy bins and compared for the three different pixel clustering methods. Later, they are assessed by selecting energies between 0-15 $\mathrm{keV}$ and comparing the result with that provided by a photoncounting detector image without energy or time information.

\section{RESULTS}

\section{A. Energy spectrum}

The detailed spectrum was obtained for the three clustering cases using the Timepix3 spectral information (see Fig. 4). The unclustered spectra show a significantly stronger lowenergy peak which is caused by charge-sharing events. The clustered spectra show that clustering is key to reconstruct high-energy events (above $15 \mathrm{keV}$ ), and that without them, limited spectroscopic information can be retrieved after 25 $\mathrm{keV}$.

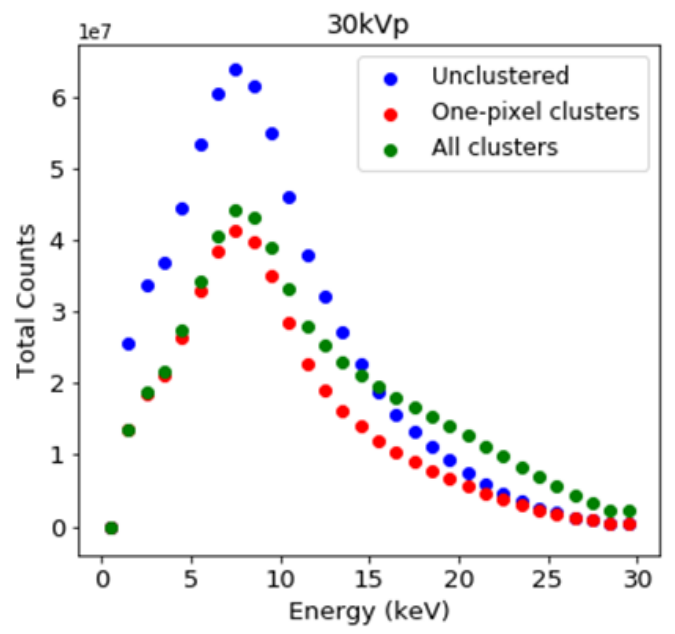

Fig. 4. Histograms of experimental spectra for unclustered, one-pixel clusters and all- clusters cases for $30 \mathrm{kVp}$.

\section{B. Clustering and spectral analysis}

The retrieved images for each of the $5 \mathrm{keV}$ bins are presented in Fig. 5a for the FSP-XPCI method. The energy dependence of the phase and absorption signals due to the 
a)

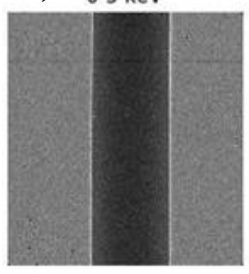

15-20 keV

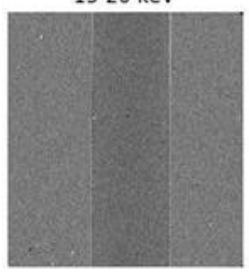

5-10 keV

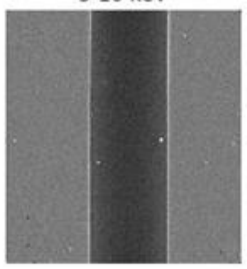

20-25 keV

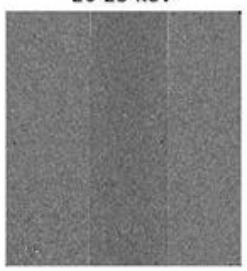

10-15 keV

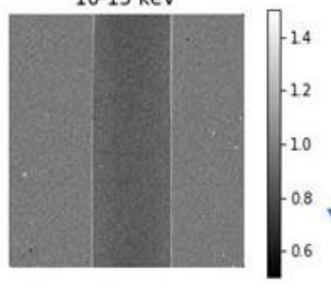

25-30 keV

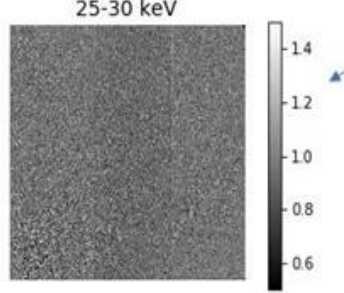

b)

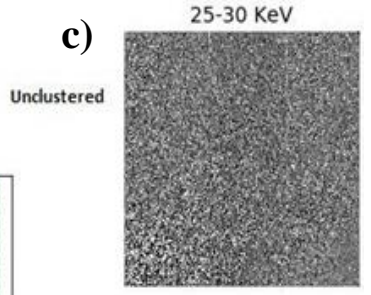

25-30 keV

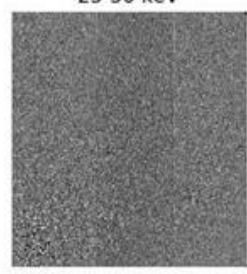

Fig. 5. a) Images retrieved for each $5 \mathrm{keV}$ energy bin at $30 \mathrm{kVp}$ with all-clusters. b) Experimental $30 \mathrm{kVp}$ spectrum with energy bins division. c)

25-30keV image comparison for unclustered and all-clusters cases.

complex refractive index coefficients is visually evident and is further demonstrated in Fig. 6. In the specific case of the sample used, low energy bins $(0-15 \mathrm{keV})$ provide most of the attenuation and edge enhancement information. In addition, note that despite the low attenuation in the 20-25 and 25-30 $\mathrm{keV}$ images, the phase signal still allows to discern the nylon fiber shape which mostly made possible by pixel clustering (see Fig. 5c).

In order to understand the effect of pixel clustering methods and the energy dependence of the phase signal, the contrast and SNR are evaluated for each energy bin as presented in Fig. 6. In the low-energy range $(0-20 \mathrm{keV})$, clustering methods allow to obtain a higher contrast which validates that the increased low-energy contribution in the unclustered spectrum is due to charge-sharing events.

By looking at the SNR data, it can be noticed that most of the SNR contribution is concentrated near the emission lines of the tungsten target, due to both higher contrast and greater number of photons.

\section{Contrast and SNR image comparison}

In order to demonstrate the capabilities of both the pixel clustering and the energy selection methods, the unclustered image is compared with the all-clusters method plus energy selection of $0-15 \mathrm{keV}$ photons (see Fig. 7). The contrast enhancement due to absorption is visually evident in the images and the edge enhancement due to phase can also be noticed in the horizontal profiles for the two XPCI techniques. For the FSP XPCI method, contrast is increased from 0.33 to 0.44. Conversely, for the SMEI XPCI method, contrast is enhanced from 0.43 to 0.55 . Note, however, that this energy selection is specific to the tungsten anode and the lowabsorbing nylon sample.

Additionally, the properties of each XPCI can also be observed. For instance, the stronger edge enhancement $(25 \%$ higher in the $0-15 \mathrm{keV}$ image) of the SMEI XPCI method compared with FSP XPCI. However, further studies are required to compare SNR and noise between the two methods.
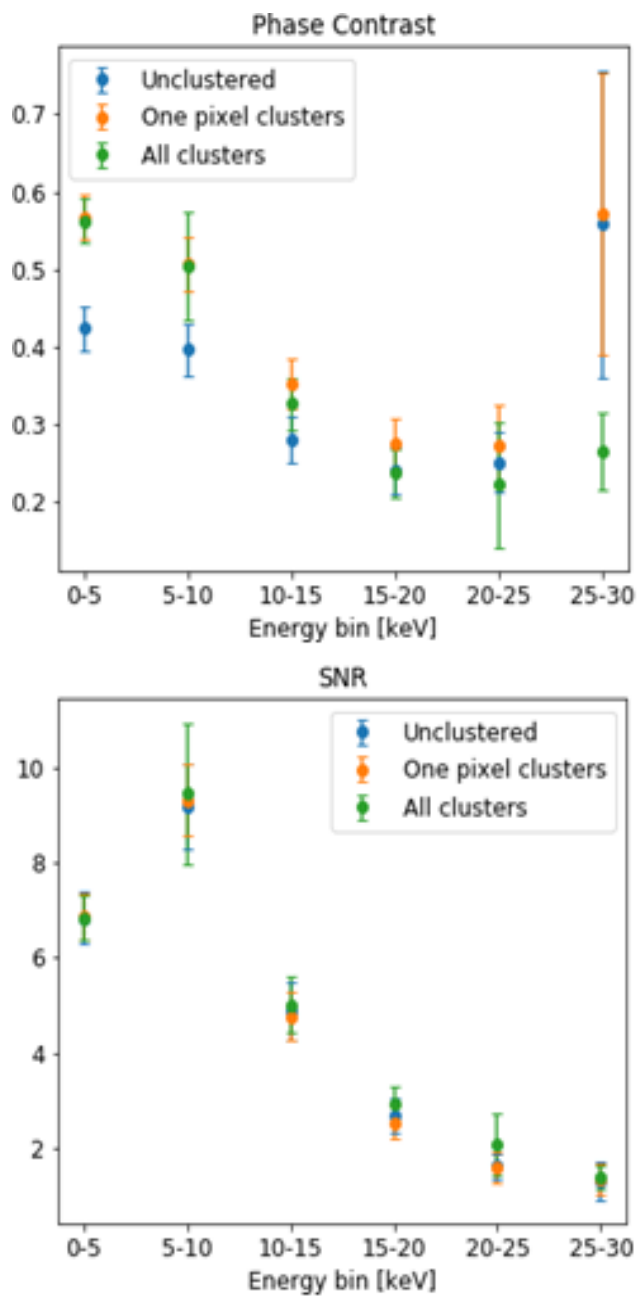

Fig. 6. Contrast and SNR as a function of the energy bin for unclustered, one-pixel clusters and all-clusters cases for the $30 \mathrm{kVp}$ image with FSP XPCI. 

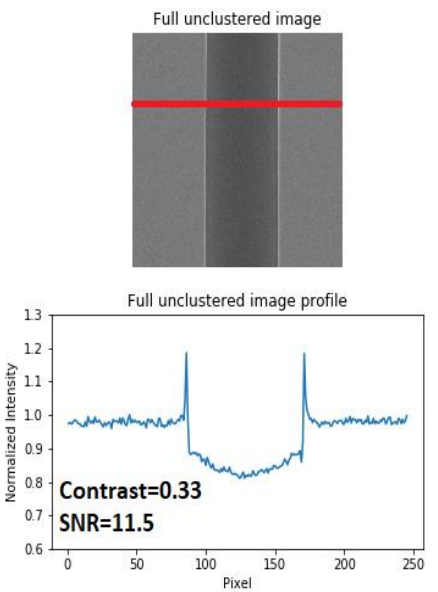

0-15 keV image

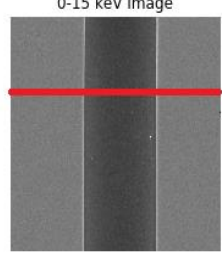

0-15 keV image profile

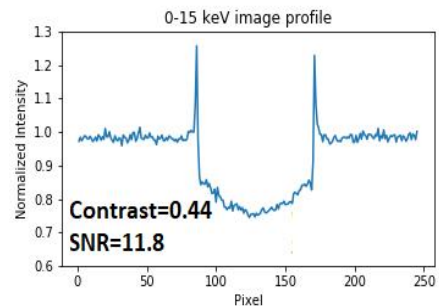

Full unclustered image

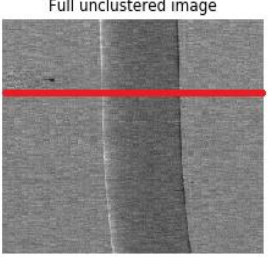

Full unclustered image profile

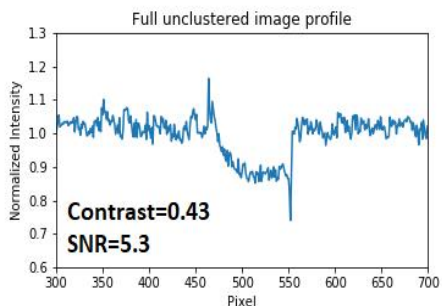

$0-15$ keV imag

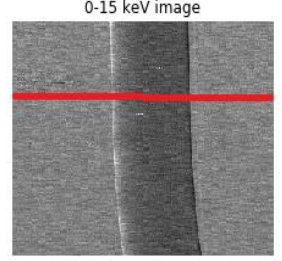

$0-15 \mathrm{keV}$ image profile

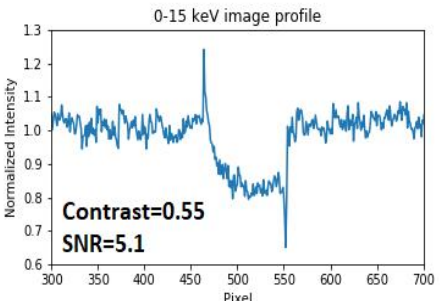

Fig. 7. Image comparison between unclustered full image and energy-selection image with all clusters for FSP XPCI and SMEI XPCI at 30kVp. The horizontal profile along the image is also shown.

\section{CONCLUSIONS}

The time and energy information of the Timepix 3 detector has the potential to improve image quality in noninterferometric XPCI techniques. Its capabilities were utilized to diminish the typical charge sharing effect in photon counting detectors and to study the energy dependence of contrast and SNR in XPCI imaging. Different clustering methods were compared and their importance in terms of effectively exploiting the physical information brought by all photons was demonstrated. Finally, an energy-window selection method was adopted and led to contrast enhancement on both XPCI methods with respect to single-threshold photon counting detectors. The combination of these two disruptive technologies could increase the capabilities of X-ray imaging.

\section{REFERENCES}

[1] R. A. Lewis, "Medical phase contrast x-ray imaging: current status and future prospects," Physics in Medicine and Biology, vol. 49, no. 16, pp. 3573-3583, 2004.

[2] S.-A. Zhou and A. Brahme "Development of phase-contrast X-ray imaging techniques and potential medical applications," Physica Medica, vol. 24, no. 3, pp. 129-148, 2008.

[3] P. Coan, A. Bravin, and G. Tromba, "Phase-contrast X-ray imaging of the breast: recent developments towards clinics," Journal of Physics D: Applied Physics, vol. 46, no. 49, p. 494007, 2013.

[4] A. Olivo, D. Chana and R. Speller, "A preliminary investigation of the potential of phase contrast $\mathrm{x}$-ray imaging in the field of homeland security", Journal of Physics D: Applied Physics, vol. 41, no. 22, p. 225503, 2008.

[5] A. Olivo, K. Ignatyev, P.R.T. Munro and R.D. Speller, "Design and realization of a coded-aperture based X-ray phase contrast imaging for homeland security applications," Nucl. Instrum. Methods Phys. Res. B, vol. 610, no. 2, pp. 604-614, 2009.

[6] A. Stevenson, T. Gureyev, D. Paganin, S. Wilkins, T. Weitkamp, A. Snigirev, et al., "Phase-contrast X-ray imaging with synchrotron radiation for materials science applications," Nucl. Instrum. Methods Phys. Res. B, vol. 199, pp. 427-435, 2003.

[7] S.C. Mayo, A.W. Stevenson and S.W. Wilkins, "In-line phase-contrast X-ray imaging and tomography for materials science," Materials, vol. 5, no. 12 , pp. 937-965, 2012.

[8] A. Olivo and E. Castelli, "X-ray phase contrast imaging: From synchrotrons to conventional sources," Riv. Nuovo Cimento, vol. 37, no. 9, pp. 467-508, 2014.
[9] A. Snigirev, I. Snigireva, V. Kohn, S. Kuznetsov, and I. Schelokov, "On energy synchrotron radiation," Review of Scientific Instruments, vol. 66, no. 12, pp. 5486-5492, 1995.

[10] S. W. Wilkins, T. E. Gureyev, D. Gao, A. Pogany, and A. W. Stevenson, "Phase-contrast imaging using polychromatic hard X-rays," Nature, vol. 384, no. 6607, pp. 335-338, 1996.

[11] P. Cloetens., S. Pateyron, M., Buffiere, J.Y., Peix, G., J. Baruchel, F. Peyrin and M. Schlenker. "Observation of microstructure and damage in materials by phase sensitive radiography and tomography," J. Appl. Phys., vol. 81, no. 9, pp. 5878-5886, 1997.

[12] S.W. Wilkins, D. Gao, T. Gureyev, A. Pogany and A.W. Stevenson. "Xray phase-contrast radiography,", Radiology, vol. 205, pp. 907-907, 1997.

[13] O. Betz, U. Wegst, D. Weide, M. Heethoff, L. Helfen, W. Lee and P. Cloetens, "Imaging applications of synchrotron X-ray phase-contrast microtomography in biological morphology and biomaterials science. General aspects of the technique and its advantages in the analysis of millimetre-sized arthropod structure," Journal of Microscopy, vol. 227, pp. 51-71, 2007.

[14] Y. Liu, J. Nelson, C. Holzner, J.C. Andrews and P. Pianetta, "Recent advances in synchrotron-based hard x-ray phase contrast imaging," Journal of Physics D: Applied Physics, vol. 46, no. 49, 2013.

[15] B. Zoofan, J.-Y. Kim, S. I. Rokhlin and G. S. Frankel, "Phase-contrast x-ray imaging for nondestructive evaluation of materials," Journal of Applied Physics, vol. 100, no. 1, p. 014502, 2006.

[16] T. Zhou, U. Lundström, T. Thüring, S. Rutishauser, D. H. Larsson, M. Stampanoni, C. David, H. M. Hertz, and A. Burvall, "Comparison of two x-ray phase-contrast imaging methods with a microfocus source", Optics Express, vol. 21, no. 25, pp. 30183-30195, 2013.

[17] A. Olivo, F. Arfelli, G. Cantatore, R. Longo, R. H. Menk, S. Pani, M. Prest, P. Poropat, L. Rigon, G. Tromba, E. Vallazza, and E. Castelli, “An innovative digital imaging set-up allowing a low-dose approach to phase contrast applications in the medical field," Medical Physics, vol. 28, no. 8, pp. 1610-1619, 2001.

[18] A. Olivo and R. Speller. A coded-aperture technique allowing X-ray phase contrast imaging with conventional sources. Applied Physics Letters, vol. 91, no, 7, p. 074106, 2007.

[19] G.K. Kallon, F. Vittoria, M. Endrizzi, P.C. Diemoz, C.K. Hagen, A. Zamir, D. Basta and A. Olivo, "Detector requirements for single mask edge illumination $\mathrm{x}$-ray phase contrast imaging applications," 11th International Conference on Position Sensitive Detectors, 2017.

[20] F. Krejci, J. Jakubek, and M. Kroupa, "Hard x-ray phase contrast imaging using single absorption grating and hybrid semiconductor pixel detector," Review of Scientific Instruments, vol. 81, no. 11, p. 113702, 2010.

[21] G K Kallon, P C Diemoz, F A Vittoria, D Basta, M Endrizzi and A Olivo. "Comparing signal intensity and refraction sensitivity of double and single mask edge illumination lab-based $\mathrm{x}$-ray phase contrast imaging set-ups", Journal of Physics D: Applied Physics, vol. 50, no. 41, 2017 
[22] T. Poikela, J. Plosila, T. Westerlund, M. Campbell, M. D. Gaspari, X. Llopart, V. Gromov, R. Kluit, M. V. Beuzekom, F. Zappon, V. Zivkovic, C. Brezina, K. Desch, Y. Fu, and A. Kruth, "Timepix3: a 65K channel hybrid pixel readout chip with simultaneous ToA/ToT and sparse readout," Journal of Instrumentation, vol. 9, no. 05, 2014.

[23] T. Billoud, C. Leroy, C. Papadatos, M. Pichotka, S. Pospisil, and J. Roux, "Characterization of a pixelated CdTe Timepix detector operated in ToT mode," Journal of Instrumentation, vol. 12, no. 01, 2017.

[24] D. Gürsoy and M. Das, "Single-step absorption and phase retrieval with polychromatic x rays using a spectral detector,". Optics Letters, vol. 38, no. 9, pp. 1461-1463, 2013.

[25] M. Das and Z. Liang, "Spectral X-ray phase contrast imaging for singleshot retrieval of absorption, phase, and differential-phase imagery," Optics Letters, vol. 39, no. 21, pp. 6343-6346, 2014.

[26] Z. Wang, K. Gao, D. Wang, Z. Wu, H. Chen, S. Wang, and Z. Wu. "Single-shot $\mathrm{x}$-ray phase imaging with grating interferometry and photon-counting detectors," Optics Letters, vol. 39, no. 4, pp. 877-879, 2014.

[27] J. Jakubek, "Precise energy calibration of pixel detector working in time-over-threshold mode," Nucl. Instrum. Meth. Phys. Res. A, vol. 633, no. 1, p. 262-266, 2011.

[28] J. Jakubek, "Energy-sensitive x-ray radiography and charge sharing effect in pixelated detector," Nucl. Instrum. Meth. Phys. Res. A, vol. 607, no. 1, p. 192-195, 2009. 